\title{
SIMULTANEOUS MAJ STATISTICS
}

\author{
Dongsu Kim and Dennis Stanton
}

ABstract. The generating function for words with several simultaneous maj weights is given. New maj-like Mahonian statistics result. Some applications to integer partitions are given.

\section{Introduction.}

The usual maj statistic [2] on words $w$ is defined by adding the location of the descents of the word $w$,

$$
\operatorname{maj}(w)=\sum_{i: w_{i}>w_{i+1}} i
$$

This definition presumes that the alphabet for the letters of $w$ have been linearly ordered, for example $2>1>0$,

$$
\operatorname{maj}(1102201)=2+5=7=\operatorname{maj}_{210}(1102201) .
$$

However a similar definition can be made assuming any linear ordering $\sigma$; here we take $1>2>0, \sigma=120$, and $2>0>1, \sigma=201$

$$
\operatorname{maj}_{120}(1102201)=2+5=7, \quad \operatorname{maj}_{201}(1102201)=5+6=11 .
$$

In this paper we consider the generating function for several such simultaneous maj statistics (see Corollary 1). A more general generating function is given (Theorem 3), and some applications to Mahonian statistics (Corollary 2) and integer partitions (Theorem 4) are stated.

We first give a 3 letter theorem, which motivates the general result (Theorem 3$)$. Let $W(m, n, k)$ be the set of words of length $m+n+k$ with $m 0$ 's, $n 1$ 's and $k$ 's.

The first author is partially supported by KOSEF: 971-0106-038-2. 
Theorem 1. For any non-negative integers $m, n$, and $k$ we have

$$
\begin{gathered}
\sum_{w \in W(m, n, k)} x^{\operatorname{maj}_{120}(w)} y^{\operatorname{maj}_{201}(w)} z^{m a j_{012}(w)}=x^{n+k} y^{k}\left[\begin{array}{c}
m+n+k-1 \\
m-1, n, k
\end{array}\right]_{x y z}+ \\
y^{k+m} z^{m}\left[\begin{array}{c}
m+n+k-1 \\
m, n-1, k
\end{array}\right]_{x y z}+z^{m+n} x^{n}\left[\begin{array}{c}
m+n+k-1 \\
m, n, k-1
\end{array}\right]_{x y z}
\end{gathered}
$$

Proof. We prove a stronger statement, that the three terms in Theorem 1 are the generating functions for the words in $W(m, n, k)$ ending in 0,1 , and 2 respectively.

We proceed by induction on $m+n+k$. If $w$ ends in a 0 , the penultimate letter must be either 0,1 or 2 . Using induction we must verify that

$$
\begin{gathered}
x^{n+k} y^{k}\left[\begin{array}{c}
m+n+k-1 \\
m-1, n, k
\end{array}\right]_{x y z}=x^{n+k} y^{k}\left[\begin{array}{c}
m+n+k-2 \\
m-2, n, k
\end{array}\right]_{x y z}+ \\
x^{m+n+k-1} y^{m+k-1} z^{m-1}\left[\begin{array}{c}
m+n+k-2 \\
m-1, n-1, k
\end{array}\right]_{x y z}+ \\
(x y)^{m+n+k-1} z^{m+n-1} x^{n}\left[\begin{array}{c}
m+n+k-2 \\
m-1, n, k-1
\end{array}\right]_{x y z},
\end{gathered}
$$

which is the well-known recurrence formula [1] for the $x y z$-trinomial coefficient.

The other two cases are verified similarly.

It should be noted that if any two of $x, y, z$ are set equal to 1 , then the usual maj generating function as a $q$-trinomial coefficient results.

\section{A 7-variable theorem.}

Theorem 1 contains three free variables, $x, y$ and $z$. In this section we generalize Theorem 1 to Theorem 2, which contains seven free variables. Then we indicate how to specialize Theorem 2 to obtain new explicit classes of Mahonian statistics on words of 0's, 1's, and 2's.

Suppose that the weights of the various possible ascents and descents in position $m+n+k-1$ of a word $w$ of $m 0$ 's, $n 1$ 's, and $k 2$ 's are given by

(wt10) $a_{0}^{m-1} a_{1}^{n} a_{2}^{k}$ for a descent 10 , (wt21) $b_{0}^{m} b_{1}^{n-1} b_{2}^{k}$ for a descent 21 , (wt20) $c_{0}^{m-1} c_{1}^{n} c_{2}^{k}$ for a descent 20 , (wt01) $d_{0}^{m} d_{1}^{n-1} d_{2}^{k}$ for an ascent 01 , (wt12) $e_{0}^{m} e_{1}^{n} e_{2}^{k-1}$ for an ascent 12 , (wt02) $f_{0}^{m} f_{1}^{n} f_{2}^{k-1}$ for an ascent 02 . 
Also suppose that the generating function for all such words $w$ has the form

$$
\begin{array}{r}
p_{0}(n, k)\left[\begin{array}{c}
m+n+k-1 \\
m-1, n, k
\end{array}\right]_{B}+p_{1}(k, m)\left[\begin{array}{c}
m+n+k-1 \\
m, n-1, k
\end{array}\right]_{B} \\
+p_{2}(m, n)\left[\begin{array}{c}
m+n+k-1 \\
m, n, k-1
\end{array}\right]_{B}
\end{array}
$$

for some base $B$, and $p_{0}(n, k)=p_{01}^{n} p_{02}^{k}, p_{1}(k, m)=p_{11}^{k} p_{12}^{m}, p_{2}(m, n)=$ $p_{21}^{m} p_{22}^{n}$. We also assume that the three terms in (2.1) correspond to the $w$ which end in 0,1 , and 2 respectively.

Thus we have 25 free variables

$$
\cup_{i=0}^{2}\left\{a_{i}, b_{i}, c_{i}, d_{i}, e_{i}, f_{i}, p_{i 1}, p_{i 2}\right\} \cup\{B\}
$$

These 25 variables are related by the three equations which we require by induction

$$
\begin{gathered}
p_{0}(n, k)\left[\begin{array}{c}
m+n+k-1 \\
m-1, n, k
\end{array}\right]_{B}=p_{0}(n, k)\left[\begin{array}{c}
m+n+k-2 \\
m-2, n, k
\end{array}\right]_{B} \\
+a_{0}^{m-1} a_{1}^{n} a_{2}^{k} p_{1}(k, m-1)\left[\begin{array}{c}
m+n+k-2 \\
m-1, n-1, k
\end{array}\right]_{B} \\
+c_{0}^{m-1} c_{1}^{n} c_{2}^{k} p_{2}(m-1, n)\left[\begin{array}{c}
m+n+k-2 \\
m-1, n, k-1
\end{array}\right]_{B}, \\
p_{1}(k, m)\left[\begin{array}{c}
m+n+k-1 \\
m, n-1, k
\end{array}\right]_{B}=p_{1}(k, m)\left[\begin{array}{c}
m+n+k-2 \\
m, n-2, k
\end{array}\right]_{B} \\
+b_{0}^{m} b_{1}^{n-1} b_{2}^{k} p_{2}(m, n-1)\left[\begin{array}{c}
m+n+k-2 \\
m, n-1, k-1
\end{array}\right]_{B} \\
+d_{0}^{m} d_{1}^{n-1} d_{2}^{k} p_{0}(n-1, k)\left[\begin{array}{c}
m+n+k-2 \\
m-1, n-1, k
\end{array}\right]_{B}, \\
p_{2}(m, n)\left[\begin{array}{c}
m+n+k-1 \\
m, n, k-1
\end{array}\right]_{B}=p_{2}(m, n)\left[\begin{array}{c}
m+n+k-2 \\
m, n, k-2
\end{array}\right]_{B} \\
+f_{0}^{m} f_{1}^{n} f_{2}^{k-1} p_{0}(n, k-1)\left[\begin{array}{c}
m+n+k-2 \\
m-1, n, k-1
\end{array}\right]_{B} \\
+e_{0}^{m} e_{1}^{n} e_{2}^{k-1} p_{1}(k-1, m)\left[\begin{array}{c}
m+n+k-2 \\
m, n-1, k-1
\end{array}\right]_{B}
\end{gathered}
$$

We do not know the general solution to the equations (2.2a-c). However, we will give the general solution to $(2.2 \mathrm{a}-\mathrm{c})$ if we make another assumption. If 
we specify that the coefficient of the second term on the the right side of (2.2a) is $B^{m-1}$ times the coefficient of the first term, and the coefficient of the third term is $B^{m+n-1}$ times the coefficient of the first term, then the $B$-trinomial recurrence relation verifies $(2.2 \mathrm{a})$. These two equations are

$$
\begin{aligned}
a_{0}^{m-1} a_{1}^{n} a_{2}^{k} p_{11}^{k} p_{12}^{m-1} & =B^{m-1} p_{01}^{n} p_{02}^{k}, \\
c_{0}^{m-1} c_{1}^{n} c_{2}^{k} p_{21}^{m-1} p_{22}^{n} & =B^{m+n-1} p_{01}^{n} p_{02}^{k} .
\end{aligned}
$$

Similarly, we assume the $B$-trinomial recurrence for $(2.2 \mathrm{~b})$ and $(2.2 \mathrm{c})$, which become

$$
\begin{aligned}
b_{0}^{m} b_{1}^{n-1} b_{2}^{k} p_{21}^{m} p_{22}^{n-1} & =B^{n-1} p_{11}^{k} p_{12}^{m} \\
d_{0}^{m} d_{1}^{n-1} d_{2}^{k} p_{01}^{n-1} p_{02}^{k} & =B^{n+k-1} p_{11}^{k} p_{12}^{m}
\end{aligned}
$$

and

$$
\begin{aligned}
f_{0}^{m} f_{1}^{n} f_{2}^{k-1} p_{01}^{n} p_{02}^{k-1} & =B^{k-1} p_{21}^{m} p_{22}^{n} \\
e_{0}^{m} e_{1}^{n} e_{2}^{k-1} p_{11}^{k-1} p_{12}^{m} & =B^{k+m-1} p_{21}^{m} p_{22}^{n}
\end{aligned}
$$

Since these equations should hold for all $m, n$ and $k$, each of these 6 equations contains 3 equations (one each in $m, n$, and $k$ ). Thus we have 18 equations in the 25 free variables, which are written in a matrix form, where the first column comes from the equations in $(2.3 \mathrm{a})$ :

$$
\left(\begin{array}{ccc}
p_{12} a_{0} & p_{21} b_{0} & f_{0} \\
a_{1} & p_{22} b_{1} & p_{01} f_{1} \\
p_{11} a_{2} & b_{2} & p_{02} f_{2} \\
p_{21} c_{0} & d_{0} & p_{12} e_{0} \\
p_{22} c_{1} & p_{01} d_{1} & e_{1} \\
c_{2} & p_{02} d_{2} & p_{11} e_{2}
\end{array}\right)=\left(\begin{array}{ccc}
B & p_{12} & p_{21} \\
p_{01} & B & p_{22} \\
p_{02} & p_{11} & B \\
B & p_{12} & p_{21} B \\
p_{01} B & B & p_{22} \\
p_{02} & p_{11} B & B
\end{array}\right)
$$

One may find the general solution to these 18 equations, leaving 7 free variables

$$
\left\{a_{0}, a_{1}, a_{2}, b_{0}, b_{1}, b_{2}, B\right\} .
$$

The explicit solutions for the remaining 18 variables are given below. The weights (wt) become (W):

(W10) $a_{0}^{m-1} a_{1}^{n} a_{2}^{k} \quad$ for a descent 10 , (W21) $b_{0}^{m} b_{1}^{n-1} b_{2}^{k} \quad$ for a descent 21 , (W20) $\left(a_{0} b_{0}\right)^{m-1}\left(a_{1} b_{1}\right)^{n}\left(a_{2} b_{2}\right)^{k} \quad$ for a descent 20, (W01) $\left(B / a_{0}\right)^{m}\left(B / a_{1}\right)^{n-1}\left(B / a_{2}\right)^{k} \quad$ for an ascent 01 , (W12) $\left(B / b_{0}\right)^{m}\left(B / b_{1}\right)^{n}\left(B / b_{2}\right)^{k-1}$ for an ascent 12 , (W02) $\left(B / a_{0} b_{0}\right)^{m}\left(B / a_{1} b_{1}\right)^{n}\left(B / a_{2} b_{2}\right)^{k-1} \quad$ for an ascent 02 , and

$$
\begin{aligned}
p_{0}(n, k) & =a_{1}^{n}\left(a_{2} b_{2}\right)^{k}, \quad p_{1}(k, m)=b_{2}^{k}\left(B / a_{0}\right)^{m}, \\
p_{2}(m, n) & =\left(B / a_{0} b_{0}\right)^{m}\left(B / b_{1}\right)^{n} .
\end{aligned}
$$


Theorem 2. The generating function of all words $w \in W(m, n, k)$ with weights given by $(W)$ is

$$
\begin{gathered}
a_{1}^{n}\left(a_{2} b_{2}\right)^{k}\left[\begin{array}{c}
m+n+k-1 \\
m-1, n, k
\end{array}\right]_{B}+b_{2}^{k}\left(B / a_{0}\right)^{m}\left[\begin{array}{c}
m+n+k-1 \\
m, n-1, k
\end{array}\right]_{B}+ \\
\left(B / a_{0} b_{0}\right)^{m}\left(B / b_{1}\right)^{n}\left[\begin{array}{c}
m+n+k-1 \\
m, n, k-1
\end{array}\right]_{B} .
\end{gathered}
$$

Theorem 1 is the special case of Theorem 2 for which $B=x y z$, $a_{0}=a_{1}=a_{2}=x$, and $b_{0}=b_{1}=b_{2}=y$ hold.

There are 7 other versions of Theorem 2 . These 8 theorems arise by independently replacing the pair of factors $\left(B^{m-1}, B^{m+n-1}\right)$ by $\left(B^{m+k-1}, B^{m-1}\right)$ in equation $(2.3 \mathrm{a}),\left(B^{n-1}, B^{n+k-1}\right)$ by $\left(B^{n+m-1}, B^{n-1}\right)$ in equation $(2.3 \mathrm{~b})$, and $\left(B^{k-1}, B^{k+m-1}\right)$ by $\left(B^{k+n-1}, B^{k-1}\right)$ in $(2.3 \mathrm{c})$. The $B$-trinomial recurrence still holds. For instance if we make a replacement in $(2.3 \mathrm{a})$,

$$
\begin{aligned}
a_{0}^{m-1} a_{1}^{n} a_{2}^{k} p_{11}^{k} p_{12}^{m-1} & =B^{m+k-1} p_{01}^{n} p_{02}^{k}, \\
c_{0}^{m-1} c_{1}^{n} c_{2}^{k} p_{21}^{m-1} p_{22}^{n} & =B^{m-1} p_{01}^{n} p_{02}^{k}
\end{aligned}
$$

then the explicit solutions to $\left(2.3 \mathrm{a}^{\prime}\right)$ and $(2.3 \mathrm{~b}-\mathrm{c})$ give the weight $\left(\mathrm{W}^{\prime}\right)$ :

(W'10) $a_{0}^{m-1} a_{1}^{n} a_{2}^{k} \quad$ for a descent 10 , $\left(\mathrm{W}^{\prime} 21\right) b_{0}^{m} b_{1}^{n-1} b_{2}^{k} \quad$ for a descent 21 , $\left(\mathrm{W}^{\prime} 20\right)\left(a_{0} b_{0}\right)^{m-1}\left(a_{1} b_{1} / B\right)^{n}\left(a_{2} b_{2} / B\right)^{k} \quad$ for a descent 20, (W'01) $\left(B / a_{0}\right)^{m}\left(B / a_{1}\right)^{n-1}\left(B^{2} / a_{2}\right)^{k}$ for an ascent 01, (W'12) $\left(B / b_{0}\right)^{m}\left(B / b_{1}\right)^{n}\left(B / b_{2}\right)^{k-1}$ for an ascent 12 , $\left(\mathrm{W}^{\prime} 02\right)\left(B / a_{0} b_{0}\right)^{m}\left(B / a_{1} b_{1}\right)^{n}\left(B^{2} / a_{2} b_{2}\right)^{k-1} \quad$ for an ascent 02 , and the corresponding theorem is the following:

Theorem 2'. The generating function of all words $w \in W(m, n, k)$ with weights given by $\left(W^{\prime}\right)$ is

$$
\begin{gathered}
a_{1}^{n}\left(a_{2} b_{2} / B\right)^{k}\left[\begin{array}{c}
m+n+k-1 \\
m-1, n, k
\end{array}\right]_{B}+b_{2}^{k}\left(B / a_{0}\right)^{m}\left[\begin{array}{c}
m+n+k-1 \\
m, n-1, k
\end{array}\right]_{B}+ \\
\left(B / a_{0} b_{0}\right)^{m}\left(B / b_{1}\right)^{n}\left[\begin{array}{c}
m+n+k-1 \\
m, n, k-1
\end{array}\right]_{B} .
\end{gathered}
$$

We do not state the remaining 6 variations here.

We can find Mahonian statistics by requiring that the generating function in Theorem 2 is the $B$-trinomial via the $B$-trinomial recurrence. There are six choices for this recurrence, one for each ordering of the 3 terms. So Theorem 2 gives a total of 6 possible Mahonian statistics, one of which 
$\left(\operatorname{maj}_{012}\right)$, is found by setting $a_{0}=a_{1}=a_{2}=b_{0}=b_{1}=b_{2}=1$. Theorem $2^{\prime}$ also gives a total of 6 possible Mahonian statistics, one of which is found by setting $a_{0}=a_{1}=b_{0}=b_{1}=b_{2}=1, a_{2}=B$. Similarly there are 6 possible Mahonian statistics for each of other 6 versions of Theorem 2, for a total of $6 \times 8=48$. Six of them are the six possible $\mathrm{maj}_{\sigma}$ statistics, the remaining 42 come in 7 classes of six each, and they are all variations on maj. Each class of size 6 consists of a $m a j$ variation, and 5 others which correspond to 5 non-trivial reorderings of $\{0,1,2\}$ of that maj variation. We give below one member of each class, eight in total.

We start with an example from Theorem $2^{\prime}$. If we set $a_{0}=a_{1}=b_{0}=$ $b_{1}=b_{2}=1, a_{2}=B$ in Theorem $2^{\prime}$, the weight $\left(\mathrm{W}^{\prime}\right)$ reduces to

$\left(\mathrm{W}^{\prime} 10\right) B^{k} \quad$ for a descent 10 ,

$\left(\mathrm{W}^{\prime} 21\right) 1$ for a descent 21,

$\left(\mathrm{W}^{\prime} 20\right) B^{-n}$ for a descent 20 ,

(W'01) $B^{m+n+k-1}$ for an ascent 01,

$\left(\mathrm{W}^{\prime} 12\right) B^{m+n+k-1} \quad$ for an ascent 12,

(W'02) $B^{m+n+k-1} \quad$ for an ascent 02 .

Note that the above weight $\left(\mathrm{W}^{\prime}\right)$ is a perturbation of $\mathrm{maj}_{012}$ involving the descents 10 and 20. We write it as $m a j_{012}+s_{0}$, where $s_{0}$ is defined in the following way. We define $s_{0}$ by giving the non-zero values at adjacent letters. One then adds these values to find $s_{0}$. It is assumed that if $w$ is truncated after the adjacent letters, $w$ has $m$ 0's, $n$ 1's, and $k 2$ 's. $s_{0}(w)$ :

(1) $k$ for an adjacent 10 ,

(2) $-n$ for an adjacent 20.

For example,

$$
s_{0}(22012110201)=-0+3-3=0 .
$$

It turns out (we do not write down the details here) that the eight statistics (including majo12 $_{012}$ ) can be defined by three independent perturbations of $\operatorname{maj}_{012}: s_{0}, s_{1}$, and $s_{2}$. For any subset $A \subset\{0,1,2\}$ put

$$
s_{A}(w)=\sum_{i \in A} s_{i}(w) .
$$

Then the eight Mahonian statistics are $m a j_{012}+s_{A}$. In fact the set $A$ indicates which replacements are made in $(2.3 \mathrm{a}-\mathrm{c})$. For instance the above $\left(\mathrm{W}^{\prime}\right)$ is $m j_{012}+s_{\{0\}}$ and if we make replacements, say in $(2.3 \mathrm{~b})$ and $(2.3 \mathrm{c})$, then the corresponding statistics will be $\operatorname{maj}_{012}+s_{\{1,2\}}$, and so on. We define $s_{1}, s_{2}$ analogously by giving the non-zero values at adjacent letters. One then adds these values to find the statistic. It is assumed that if $w$ is truncated after the adjacent letters, $w$ has $m 0$ 's, $n$ 1's, and $k 2$ 's. $s_{1}(w)$ :

(1) $m$ for an adjacent 21 , 
(2) $-k$ for an adjacent 01 .

$s_{2}(w)$ :

(1) $n$ for an adjacent 02 ,

(2) $-m$ for an adjacent 12 .

For example,

$s_{1}(22012110201)=-2+1-4=-5, \quad s_{2}(22012110201)=-1+3=2$.

Below is a table evaluating $\operatorname{maj}_{012}, s_{0}, s_{1}$, and $s_{2}$ at the 6 permutations of 012. Note that the $\operatorname{maj}_{012}$ generating function is $1+2 B+2 B^{2}+B^{3}$, which is also the generating function for $m a j_{012}+s_{A}$, for any subset $A \subset\{0,1,2\}$.

$\begin{array}{ccrrr}\text { word } & \operatorname{maj}_{012} & s_{0} & s_{1} & s_{2} \\ 012 & 3 & 0 & 0 & -1 \\ 021 & 1 & 0 & 1 & 0 \\ 102 & 2 & 0 & 0 & 1 \\ 120 & 1 & -1 & 0 & 0 \\ 201 & 2 & 0 & -1 & 0 \\ 210 & 0 & 1 & 0 & 0\end{array}$

We repeat that all 48 Mahonian statistics may be found from these 8 by permuting the letters 0,1 , and 2 . In this case $\operatorname{maj}_{012}$ becomes $\operatorname{maj}_{\sigma}$, and each $s_{i}$ is found by applying $\sigma$ to 0,1 , and 2 in the definition of $s_{i}$.

\section{3. $N$ letters.}

In this section we briefly generalize Theorem 2 to words with $N$ letters in Theorem 3. We state the $N$ letter version of Theorem 1 in Corollary 1. There are $N ! 2^{N}$ Mahonian statistics, which come in $2^{N}$ families each of size $N$ !. We explicitly give the corresponding $2^{N}$ Mahonian statistics in Corollary 2.

Let $W\left(a_{0}, a_{1}, \cdots, a_{N-1}\right)$ be the set of all words $w$ with $a_{i} i$ 's, $0 \leq i \leq$ $N-1$.

If the words $w$ have $N$ letters instead of 3 letters, then each adjacent pair $i j, i \neq j$, could be weighted by $N$ variables, instead of 3 variables. Also the coefficients $p_{i}, 0 \leq i \leq N-1$ would have $N-1$ variables. Together with the base $B$, we have a total of $N\left(N^{2}-N\right)+N(N-1)+1=N^{3}-N+1$ variables. Each of the $N$ recurrences required by induction gives $N(N-1)$ equations in these variables. So $N(N-1)+1$ variables will be free in the multivariable version of Theorem 2 .

In order to fully describe the resulting theorem, some care must be taken with notation.

The $N(N-1)+1$ free variables may be taken to be the base $B$ along with the $N$ weights of the adjacent pairs $(i+1) i$, for $i=0, \cdots, N-2$, for which we use the variables

$$
\left(x_{i 0}, x_{i 1}, \cdots, x_{i N-1}\right), \quad 0 \leq i \leq N-2 .
$$


Suppose that $w$ ends in an adjacent pair $i j, i \neq j$, and that there are $n_{k}$ $k$ 's preceding the last letter $j$ of $w$. The weight of the pair $i j$ is given by

$$
\begin{aligned}
& \prod_{k=0}^{N-1}\left(\prod_{l=j}^{i-1} x_{l k}\right)^{n_{k}} \quad \text { if } j<i \\
& \prod_{k=0}^{N-1}\left(B / \prod_{l=i}^{j-1} x_{l k}\right)^{n_{k}} \quad \text { if } i<j .
\end{aligned}
$$

As usual, we multiply the weights of adjacent pairs to find the weight of the word $w$.

Theorem 3. The generating function of all words $w \in W\left(a_{0}, a_{1}, \cdots, a_{N-1}\right)$ with weights given by (4.2) is

$$
\sum_{i=0}^{N-1} p_{i}\left(a_{0}, a_{1}, \cdots, a_{N-1}\right)\left[\begin{array}{c}
a_{0}+\cdots+a_{N-1}-1 \\
a_{0}, \cdots, a_{i}-1, \cdots, a_{N-1}
\end{array}\right]_{B}
$$

where

$$
p_{i}\left(a_{0}, a_{1}, \cdots, a_{N-1}\right)=\left(\prod_{l=0}^{i-1}\left(B / \prod_{k=1}^{i-l} x_{i-k, l}\right)^{a_{l}}\right)\left(\prod_{l=i+1}^{N-1}\left(\prod_{k=0}^{l-i-1} x_{i+k, l}\right)^{a_{l}}\right) .
$$

Note that $p_{i}$ in Theorem 3 is independent of $a_{i}$.

The multivariable version of Theorem 1 occurs if

$$
x_{i 0}=x_{i 1}=\cdots=x_{i N-1}=x_{i}, \quad 0 \leq i \leq N-2,
$$

and $B=x_{0} x_{1} \cdots x_{N-1}$. Then the weights (4.2) become

$$
\begin{array}{ll}
\left(x_{j} \cdots x_{i-1}\right)^{n_{0}+\cdots+n_{N-1}} & \text { if } j<i \\
\left(x_{0} \cdots x_{i-1} x_{j} \cdots x_{N-1}\right)^{n_{0}+\cdots+n_{N-1}} & \text { if } i<j
\end{array}
$$

and the next corollary holds.

Corollary 1. We have

$$
\begin{gathered}
\sum_{w \in W\left(a_{0}, \cdots, a_{N-1}\right)} \prod_{i=0}^{N-1} x_{i}^{m a j_{i+1 \cdots(N-1) 01 \cdots i}(w)}= \\
\sum_{i=0}^{N-1} p_{i}\left(a_{0}, a_{1}, \cdots, a_{N-1}\right)\left[\begin{array}{c}
a_{0}+\cdots+a_{N-1}-1 \\
a_{0}, \cdots, a_{i}-1, \cdots, a_{N-1}
\end{array}\right]_{x_{0} \cdots x_{N-1}}
\end{gathered}
$$


where

$p_{i}\left(a_{0}, a_{1}, \cdots, a_{N-1}\right)=\left(\prod_{l=0}^{i-1}\left(x_{0} \cdots x_{l-1} x_{i} \cdots x_{N-1}\right)^{a_{l}}\right)\left(\prod_{l=i+1}^{N-1}\left(x_{i} \cdots x_{l-1}\right)^{a_{l}}\right)$.

We next give the $2^{N}$ Mahonian statistics which follow from Theorem 3 . Again they may be classified by perturbations of $\operatorname{maj}_{01 \cdots N-1}$. For any subset $A \subset\{0,1, \cdots, N-1\}$, define

$$
s_{A}(w)=\sum_{i \in A} s_{i}(w)
$$

The individual statistics $s_{i}(w)$ only depend upon the subwords of $w$ ending in $i$, as in $\S 2$. For any given $i \in w$, suppose that $i$ is preceded by $n_{j} j$ 's, $0 \leq j \leq N-1$. Extend the definition of $n_{j}$ to be periodic $\bmod N: n_{j+N}=n_{j}$ for all $j$. If the letter preceding $i$ is $i+k$, the contribution to $s_{i}(w)$ is positive on the circular interval $[i+k+1, i-1]$ and negative on the circular interval $[i+1, i+k-1]$

$$
\left(n_{i+k+1}+n_{i+k+2}+\cdots+n_{(i-1)}\right)-\left(n_{i+1}+n_{i+2}+\cdots+n_{i+k-1}\right) .
$$

We add the contributions of (3.1) over all $i \in w$ to find $s_{i}(w)$. There is no contribution if $k=0$; that is, for a repeated $i i$. For example,

$s_{1}(41241012411312301)=0+(-1)+(-3)+(1-2)+(4-2)+(-8)=-11$.

Corollary 2. For any set $A \subset\{0,1, \cdots, N-1\}$, the statistic maj $j_{01 \cdots N-1}+$ $s_{A}$ is Mahonian on $W\left(a_{0}, a_{1}, \cdots, a_{N-1}\right)$.

These Mahonian statistics are examples of splittable statistics [3].

One may also allow weights on the adjacent letters 00, 11, and 22 for a more general version of Theorem 3 .

\section{Applications to partitions.}

In this section we apply Theorem 1 and Theorem 3 to integer partitions.

The special case $k=0, z=1, x=y=q$ of Theorem 1 is

$$
\sum_{w \in W(m, n, 0)} q^{m a j_{10}(w)+m a j_{01}(w)}=\left[\begin{array}{c}
m+n \\
m
\end{array}\right]_{q^{2}} \frac{q^{m}+q^{n}}{1+q^{m+n}}:=f(m, n, q) .
$$

MacMahon [4, p. 139] previously gave (4.1).

The following generating function (using standard notation found in [1]) follows from (4.1),

$$
\sum_{m, n \geq 0} f(m, n, q) \frac{(x q)^{m}(y q)^{n}}{(q ; q)_{m+n}}=\frac{\left(x y q^{2} ; q^{2}\right)_{\infty}}{(x q, y q ; q)_{\infty}}
$$


One way to see (4.2) is to consider the generating function for pairs of partitions $(\lambda, \mu)$ with distinct parts, weighted by

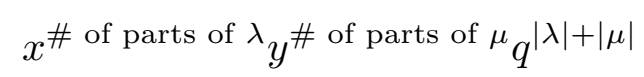

which is

$$
\prod_{k=1}^{\infty}\left(1+\frac{x q^{k}}{1-x q^{k}}+\frac{y q^{k}}{1-y q^{k}}\right)=\frac{\left(x y q^{2} ; q^{2}\right)_{\infty}}{(x q, y q ; q)_{\infty}} .
$$

To prove (4.1), we must find a weight preserving bijection $\phi$ from the set of such $(\lambda, \mu)$, \# parts of $\lambda=m$, \# parts of $\mu=n$, to the set of ordered pairs $(w, \gamma)$, where $w \in W(m, n, 0)$, and $\gamma$ is a partition with $m+n$ parts.

To define $w$, order the $m+n$ parts of $\lambda \cup \mu$ into a partition $\theta$, and let $w_{i}=0$ if $\theta_{i} \in \lambda, w_{i}=1$ if $\theta_{i} \in \mu$. This is well defined since the parts of $\lambda$ and $\mu$ are distinct. To define $\gamma$, let $t_{i}$ be the number of descents or ascents to the right of position $i$ in the word $w$. Then we let $\gamma=\theta-t$. For example if

$$
\lambda=7742, \quad \mu=88661
$$

then

$$
\theta=887766421, \quad w=110011001, \quad t=443322110, \quad \gamma=444444311
$$

This correspondence is the desired bijection $\phi$.

The natural analog of $\phi$ on triples $(\lambda, \mu, \theta)$ without pairwise common parts produces a word $w \in W(m, n, k)$ and a partition $\gamma$. The $q$-statistic on the word $w$ again counts all ascents and descents of $w$ by their positions. However, in Theorem 1, we see that the six possible ascents/descents in $w$ are weighted differently by position:

01 by $y z$,

02 by $z$,

10 by $x$,

12 by $x z$,

20 by $x y$,

21 by $y$.

So if we choose $x=q^{a}, y=q^{b}, z=q^{c}$, an occurrence of 01 in positions $j$ and $j+1$ of $w$ contributes a weight of $q^{j(b+c)}$. This in turn implies that the bijection $\phi$ must be modified so that the part in $\lambda$ corresponding to $w_{j}$ must be at least $b+c$ larger than the part in $\mu$ corresponding to $w_{j+1}$. We need six different inequalities for the six possible juxtapositions of parts. Let $\phi_{a, b, c}$ be the modified bijection. 
For example, if $m=k=2, n=1, a=2, b=c=1$, then the juxtaposed parts sizes must differ by

$$
\begin{aligned}
& 2 \text { for } \lambda \mu, \\
& 1 \text { for } \lambda \theta, \\
& 2 \text { for } \mu \lambda, \\
& 3 \text { for } \mu \theta, \\
& 3 \text { for } \theta \lambda, \\
& 1 \text { for } \theta \mu .
\end{aligned}
$$

The three possible triples $(\lambda, \mu, \theta)$ whose weight is $q^{12}$ are given below, along with result of the bijection $\phi_{2,1,1}$ :

$$
\begin{aligned}
& (22,6,11) \rightarrow(10022,31111), \\
& (32,5,11) \rightarrow(10022,22111), \\
& (43,1,22) \rightarrow(00221,21111) .
\end{aligned}
$$

Corollary 3. Let $a, b$ and $c$ be positive integers. The generating function for all triples of partitions $(\lambda, \mu, \theta)$ without pairwise common parts, such that $\lambda$ has $m$ parts, $\mu$ has $n$ parts, and $\theta$ has $k$ parts, and any adjacent parts in the partition $\lambda \cup \mu \cup \theta$ of type

(1) $\lambda \mu$ differ by $b+c$,

(2) $\lambda \theta$ differ by $c$,

(3) $\mu \lambda$ differ by $a$,

(4) $\mu \theta$ differ by $a+c$,

(5) $\theta \lambda$ differ by $a+b$,

(6) $\theta \mu$ differ by $b$,

is given by

$$
\begin{aligned}
& \frac{q^{m+n+k}}{(q ; q)_{m+n+k}}\left(q^{a(n+k)+b k}\left[\begin{array}{c}
m+n+k-1 \\
m-1, n, k
\end{array}\right]_{q^{a+b+c}}^{+}\right. \\
& \left.q^{b(m+k)+c m}\left[\begin{array}{c}
m+n+k-1 \\
m, n-1, k
\end{array}\right]_{q^{a+b+c}}+q^{c(n+m)+a n}\left[\begin{array}{c}
m+n+k-1 \\
m, n, k-1
\end{array}\right]_{q^{a+b+c}}\right) .
\end{aligned}
$$

In Theorem 3, if all $x_{i}=q$, the following theorem results. All subscripts are taken $\bmod N$.

Theorem 4. The generating function for all $N$-tuples of integer partitions $\left(\lambda_{1}, \cdots, \lambda_{N}\right)$ without pairwise common parts, such that

(a) $\lambda_{i}$ has $a_{i}$ parts, $1 \leq i \leq N$,

(b) if the partition $\lambda_{1} \cup \lambda_{2} \cup \cdots \cup \lambda_{N}$ has adjacent parts bc, for $b \in \lambda_{i}$ and $c \in \lambda_{j}$, then $b-c \geq(i-j) \bmod N$, 
is given by

$$
\frac{q^{f}}{(q ; q)_{f}}\left[\begin{array}{c}
a_{1}+\cdots+a_{N} \\
a_{1}, \cdots, a_{N}
\end{array}\right]_{q^{N}} \frac{\sum_{i=1}^{N} q^{e_{i}}}{\sum_{i=0}^{N-1} q^{i f}},
$$

where $f=a_{1}+a_{2}+\cdots+a_{N}$, and $e_{i}=a_{i}+2 a_{i+1}+\cdots+(N-1) a_{i+N-2}$.

\section{Remarks.}

MacMahon [5, §30] defined a statistic related to maj, denoted here by $M A J$, which weights each descent by the amount of the descent. For example,

$$
M A J(20211201)=2 * 1+1 * 3+2 * 6=17,
$$

because the descent 20 in positions 1,6 are weighted by $2-0=2$, while the descent 21 in position 3 is weighted by $2-1=1$. Let $M I N$ denote the analogous statistic using the ascents. Then MacMahon alludes [5, §40] to the following theorem for words with three letters.

Theorem 5. For any non-negative integers $m, n$, and $k$ we have

$$
\begin{gathered}
\sum_{w \in W(m, n, k)} x^{M A J(w)} y^{M I N(w)}=x^{n+2 k}\left[\begin{array}{c}
m+n+k-1 \\
n
\end{array}\right]_{x y}\left[\begin{array}{c}
m+k-1 \\
m-1
\end{array}\right]_{(x y)^{2}} \\
+y^{m-k}\left[\begin{array}{c}
m+n+k-1 \\
n-1
\end{array}\right]_{x y}\left[\begin{array}{c}
m+k \\
m
\end{array}\right]_{(x y)^{2}} \frac{(x y)^{2 k}+(x y)^{m+k}}{1+(x y)^{m+k}} \\
+y^{2 m+n}\left[\begin{array}{c}
m+n+k-1 \\
n
\end{array}\right]_{x y}\left[\begin{array}{c}
m+k-1 \\
m
\end{array}\right]_{(x y)^{2}} .
\end{gathered}
$$

If $x=y, y=1$ or $x=1$, the three terms in Theorem 5 sum to a single product (see $[5, \S 38, \S 40])$. The proof of Theorem 5 is identical to the proof of Theorem 1. We do not know a multivariable version of Theorem 5 .

\section{REFERENCES}

1. G. Andrews, The Theory of Partitions, Addison-Wesley, Reading, 1976.

2. D. Foata and M.-P. Schützenberger, Major index and inversion number of permutations, Math. Nachr. 83 (1978), 143-158.

3. J. Galovich and D. White, Recursive statistics on words, Disc. Math. 157 (1996), 169-191.

4. P. MacMahon, Combinatory Analysis (3rd, ed.), Chelsea, New York, 1984.

5. - The indices of permutations and the derivation therefrom of functions of a single variable associated with the permutations of any assemblage of objects, Amer. J. Math. 35 (1913), 281-322.

Department of Mathematics, KAist, Taejon 305-701, Korea

School of Mathematics, University of Minnesota, Minneapolis, MN 55455

E-mail address: dskim@math.kaist.ac.kr stanton@math.umn.edu 